\title{
Improving data access and use for HIV biomedical prevention interventions
}

Project SOAR

Follow this and additional works at: https://knowledgecommons.popcouncil.org/departments_sbsr-hiv

Part of the Health Services Research Commons How does access to this work benefit you? Let us know!

\section{Recommended Citation}

Project SOAR. 2021. "Improving data access and use for HIV biomedical prevention interventions," Learnings from Project SOAR, Synthesis Brief. Washington, DC: Population Council. 


\section{Improving Data Access and Use for HIV Biomedical Prevention Interventions}

\section{Learnings from Project SOAR}

Over the past decade, biomedical prevention interventions-including voluntary medical male circumcision (VMMC) and oral pre-exposure prophylaxis (PrEP)-have resulted in substantial gains in controlling the global HIV epidemic. Despite the inclusion of these highly effective interventions in multi-national strategies and global prevention toolkits, their effective implementation rests upon context-specific, data-driven delivery to priority populations and geographies.

Project SOAR recognizes the key role that VMMC and PrEP services play in achieving UNAIDS' 90-9090 goals for controlling the HIV epidemic through testing, treatment, and viral suppression by 2020. Drawing on its extensive portfolio, Project SOAR has contributed to the evidence base by increasing access to and use of data through user-friendly interfaces and dissemination strategies, advancing VMMC and PrEP as vital prevention interventions in the global discourse. SOAR's biomedical prevention portfolio has supported data-driven planning, implementation, and monitoring of VMMC and/or PrEP programs and policies in 10 countries-Eswatini, Kenya, Lesotho, Malawi, Mozambique, Namibia, South Africa, Tanzania, Uganda, and Zimbabwe.

This brief synthesizes results from 10 countries (see map on next page for additional details) that advanced:

- Modeling and costing for data-driven PrEP and VMMC programming.

- VMMC tool development, dissemination, and use.

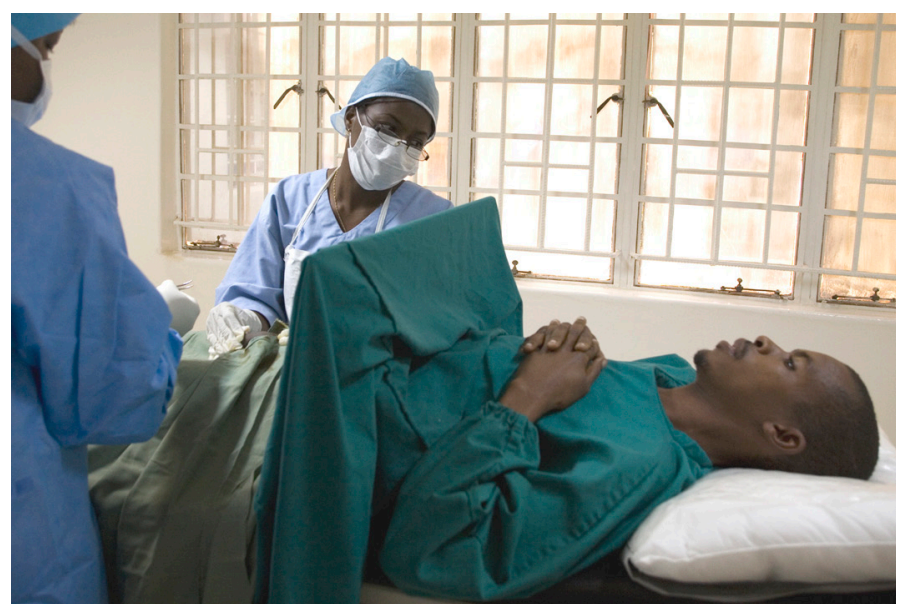

\section{KEY RECOMMENDATIONS}

1. Expand use of modeling tools and costing analyses to enhance HIV prevention through data-driven VMMC and PrEP programming. SOAR's estimates of priority population size, geographic and age-related targets, costs, and associated intervention impacts have provided countries with powerful data to target resources and strengthen biomedical prevention interventions.

2. Increase capacity for sustained data use by equipping countries with accessible and user-friendly tools, such as the VMMC suite of tools, to facilitate strategic datafor-decision-making. Close collaboration with country teams in introducing and applying these tools promotes data-driven policy and program responses. 


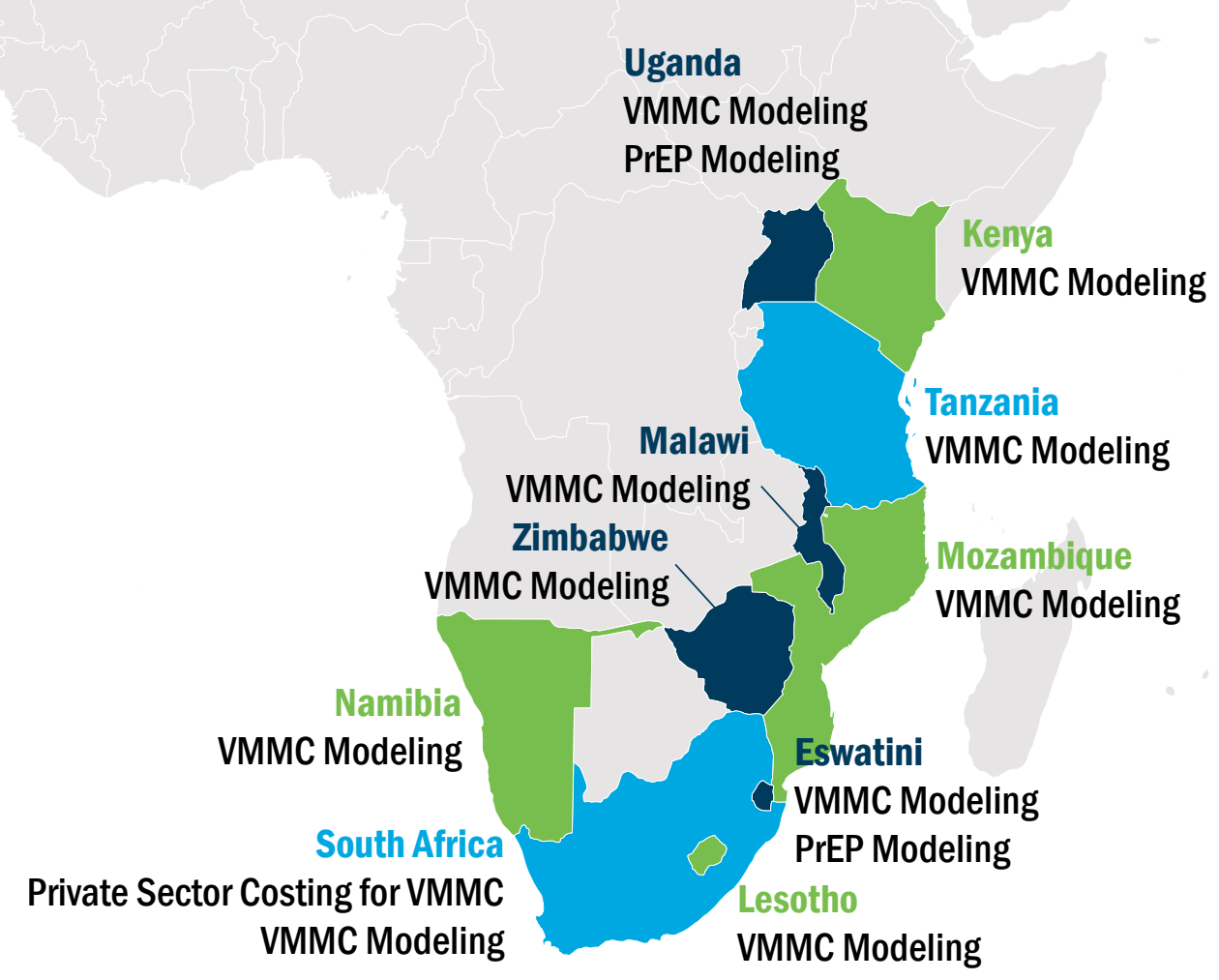

\section{ABOUT}

Project SOAR (Supporting Operational AIDS Research), was a 6-year USAID-funded implementation science initiative with over 70 activities in 21 countries. The Population Council led Project SOAR in collaboration with Avenir Health, Elizabeth Glaser Pediatric AIDS Foundation (EGPAF), Johns Hopkins University, Palladium, and The University of North Carolina at Chapel Hill.

\section{Research Utilization}

Project SOAR implemented a systematic and proactive research utilization (RU) approach to advance translation of research results into evidence-informed practice. This $\mathrm{RU}$ approach supported inclusion of end data users in the research process from inception to dissemination and fostered data-driven policy and program change.

\section{Series}

"Learnings from Project SOAR" seeks to highlight key results, recommendations, and examples of research impact across this 6-year, 21-country initiative.

The series includes five thematic briefs focused on biomedical prevention interventions, HIV testing services, HIV treatment strategies, stigma, and research utilization and capacity strengthening.

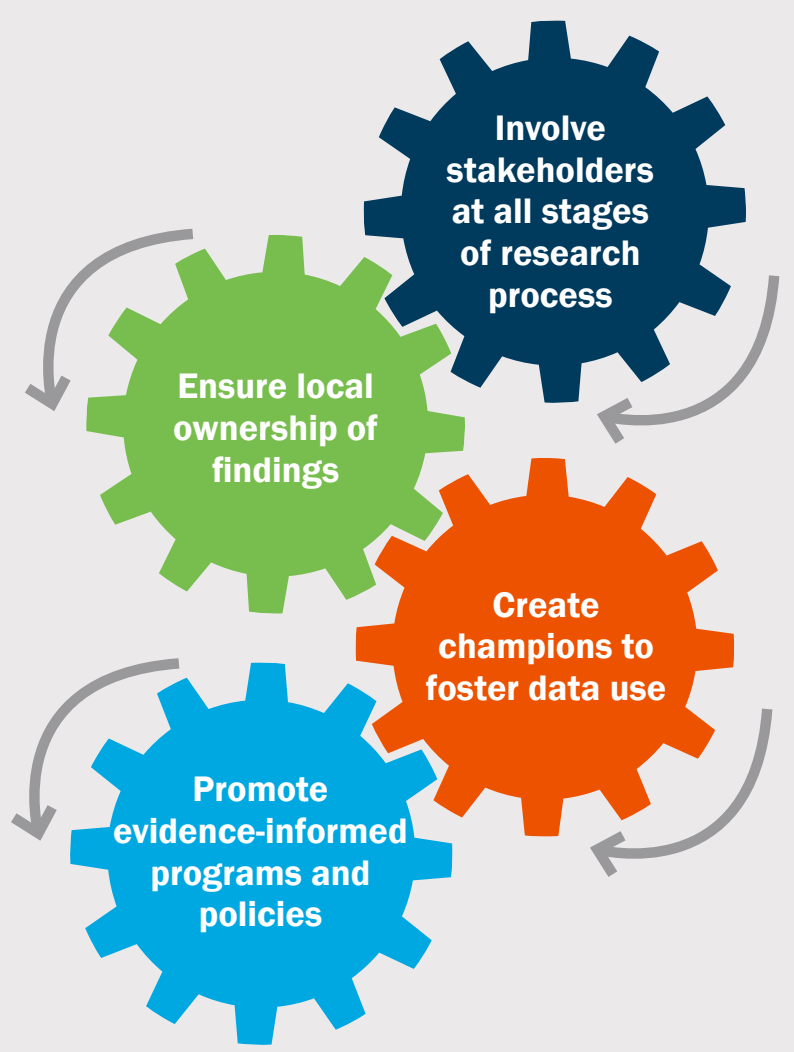




\section{MODELING AND COSTING FOR DATA-DRIVEN PREP AND VMMC PROGRAMMING}

Overview: Recent analyses in several sub-

Saharan African countries have prompted

reexamination of the target populations and

subnational regions for national biomedical

prevention strategies and operational plans. To

assist governments in the strategic planning

and program monitoring, Project SOAR

conducted modeling and costing analyses

to provide impact and target population size

estimates-as well as program cost, cost per

infection averted, and cost-effectiveness-

disaggregated by age and province or district.

Results summary: Modeling is a useful PrEP

and VMMC policy and program planning tool to

determine which populations, age subgroups,

and geographic areas offer the most potential

to efficiently achieve significant reductions in

HIV incidence in terms of scale up and cost

savings. In addition, cost analyses can identify

potential opportunities for increasing access

to services and informing policy dialogue on

reimbursement rates.

\section{Key results}

In Eswatini and Uganda, Project SOAR worked with country stakeholders to identify and answer specific modeling questions to help advance national PrEP policy and implementation planning.

Oral PrEP is a key component of PEPFAR's HIV prevention toolkit, and prior modeling work has demonstrated that targeting populations at very high risk of infection is the most cost-effective PrEP strategy. ${ }^{1}$ In Eswatini, the Project SOAR team worked with stakeholders to identify specific modeling questions to advance national PrEP policy and implementation planning. SOAR then adapted, expanded, and applied existing modeling tools (Goals
Model and Incidence Patterns Model ${ }^{2}$ ) to estimate the expected cost-effectiveness and impact of oral PrEP within the country's larger portfolio of HIV prevention interventions. This modeling exercise prompted the Ministry of Health ( $\mathrm{MOH}$ ) to offer PrEP to the general population of adult men aged 25 years and older based on the epidemiology of Eswatini's HIV epidemic. At the request of the $\mathrm{MOH}$, SOAR also conducted additional analyses to equip the $\mathrm{MOH}$ with data to make the case for PrEP to the Ministry of Finance. ${ }^{3}$

In Uganda, SOAR's modeling exercise-conducted with the $\mathrm{MOH}, \mathrm{U}$. S. Agency for International Development (USAID)/Uganda, and other stakeholders-determined that while providing oral PrEP to female sex workers and sero-discordant couples was the most costeffective strategy, including medium-risk adolescent girls and young women (AGYW) as well would increase the impact of the program in terms of reducing new HIV infections. SOAR also found that geographically focusing oral PrEP for AGYW on higher incidence areas would improve the cost-effectiveness of the program. These findings confirmed and reinforced the plans that the $\mathrm{MOH}$ had been developing for oral PrEP roll-out. In addition, SOAR's stakeholder consultations conducted in both countries informed the development of an analytical framework that was applied to an additional nine countries. ${ }^{4}$

\section{In Namilbia, modeling results supported renewed policy dialogue around targeted circumcision provision, leading to dramat- ic increases in VMMC coverage for 10- to 14-year-olds.}

Prior to 2016, Namibia's VMMC program focused almost exclusively on clients aged 15- to 29-yearsold. SOAR's modeling showed how increased VMMC service provision to younger boys, aged 10-14 years, would substantially expand coverage within a group relevant to HIV risk reduction. While clients from this younger age group were presenting for services at public facilities, they were generally turned away and referred to private providers. This referral pattern emerged from a common misinterpretation of existing Ministry of Health and Social Services policy regarding which providers were permitted to offer the procedure. Referrals of these younger clients were rarely followed up on, and either the boys did not access services, 
or they were not tracked. SOAR's analysis prompted program managers and implementers to reconsider and reinterpret existing policies to allow nurses to be trained, and for public facilities to serve younger clients. This renewed policy dialogue increased the circumcision rate of 10- to 14-year-olds by more than 20 -fold the year after the modeling exercise. ${ }^{5}$

\section{In South Africa, costs of providing VMMC in the private and public sectors are similar, offering the potential to increase access to services and inform discussions about reim- bursement rates.}

Program costing is a common data gap, and costing analyses provide important insights that can inform policy dialogue and program strategies. For instance, private sector providers offer the potential to increase access to VMMC services, as they attract clients who may not necessarily go to public facilities for services, thereby reaching pockets of the population that may have otherwise gone unreached. The private sector unit cost for VMMC service provision, however, and the cost variation across the private sector are not well known. In South Africa, Project SOAR responded to this gap by conducting a costing analysis of VMMC service provision at 10 private sector facilities in three provinces (Gauteng, Mpumalanga, and KwaZulu Natal) as a follow on to a previous costing study of VMMC through the country's public facilities. The SOAR study found that the private sector VMMC unit cost (\$137) did not differ substantially from that at government and PEPFAR-supported facilities (\$132). The two largest cost drivers, consumables and direct labor, were also comparable. Results from this study are informing discussions with private insurance providers in South Africa about standardization of VMMC tariffs. They also provide a strong rationale for reimbursing private sector providers for circumcisions of uninsured clients. ${ }^{6}$

\section{VMMC TOOL DEVELOPMENT, DISSEMINATION, AND CAPACITY STRENGTHENING}

Overview: Ministries of health, national

AIDS control programs, PEPFAR teams, and

implementers require user-friendly methods for

setting VMMC targets and planning programs

based on epidemiological data. While countries

have numerous sources of data, they often lack

structured ways of synthesizing and visualizing

these data to understand trends and adapt

program implementation accordingly. Since

2014, Project SOAR has been at the forefront

of the current phase of VMMC modeling

focused on development and dissemination of

a suite of tools to provide context-specific data

to inform VMMC program, policy, and strategic

planning decisions, including at national and

sub-national levels.

Results summary: Project SOAR and its

partners developed, disseminated, and

strengthened capacity for use of three

online VMMC data-driven tools for decision

making, which can be used independently or

together, to inform the policy environment

and to strengthen the targeting and delivery

of services from national to subnational to

facility levels. Several of these tools have been

identified as best practices, having assisted

VMMC stakeholders to better focus their

demand creation and service delivery efforts

where they are most needed and will be most impactful.

4 Improving data access and use for HIV biomedical prevention interventions 


\section{Key results}

Project SOAR and partners have developed three accessible and informative data-for-decision making tools to drive VMMC program performance: the Decision Makers Program Planning Toolkit, the Site Capacity and Productivity Assessment Tool, and the VMMIC Geospatial Information System Dashboard. ${ }^{7}$

The Decision Makers' Program Planning Toolkit (DMPPT), originally developed by SOAR partner Avenir Health in 2009, provided national estimates of VMMC impact and cost-effectiveness. In 2013, a second model (DMPPT 2.0), also developed by Avenir Health, provided in-depth country-specific data for program planning, focusing specifically on age and geographic prioritization. Under Project SOAR in 2017, Avenir Health converted the 2013 DMPPT 2.0 model into a user-friendly online version, the DMPPT 2 Online, which allows country teams to generate coverage estimates and projected targets for five-year age bands at national and district levels to achieve overall VMMC objectives. The tool allows users to set a variety of target scenarios and to download Excelbased output tables that present the specific agedisaggregated targets, coverage levels, VMMC uptake rate, impact estimates (HIV infections averted), and increases in overall VMMC coverage.

The Site Capacity and Productivity Assessment Tool (SCPT) was developed by Avenir Health under Project SOAR. The SCPT is an online resource that collects monthly VMMC facility-level data to support programs to assess the productivity and technical efficiency of VMMC sites by converting program inputs (e.g., number of staff, commodities) into program outputs (e.g., circumcisions performed, utilization of facilities). It aids in improving the implementation efficiency of VMMC programs by evaluating if programmatic resources have been allocated strategically to meet program objectives/targets, as well as assess where potenital resource gaps may exist.

The VMMC Geospatial Information System (GIS) Dashboard was developed by SOAR partner Palladium. The dashboard leverages available geo-coded VMMC data to enable easy visualization and mapping of VMMC data, including data from the DMPPT 2 Online and other country sources as requested and made available by country programs. The VMMC GIS Dashboard displays available national and sub-national data on important program indicators, including VMMC coverage, unmet need, HIV prevalence and incidence, and layers this data with other demographic and geographic information, such as population by age and road networks. Originally, the VMMC GIS Dashboard was designed and piloted in partnership with stakeholders in South Africa; it was then expanded to include data for nine other priority VMMC countries. The VMMC GIS Dashboard is hosted online, allowing users of any technical level to easily access, map, and interpret VMMC data geospatially for more effective programming.

The VMMC suite of decision-making tools enable program planners to answer key questions for program scale-up
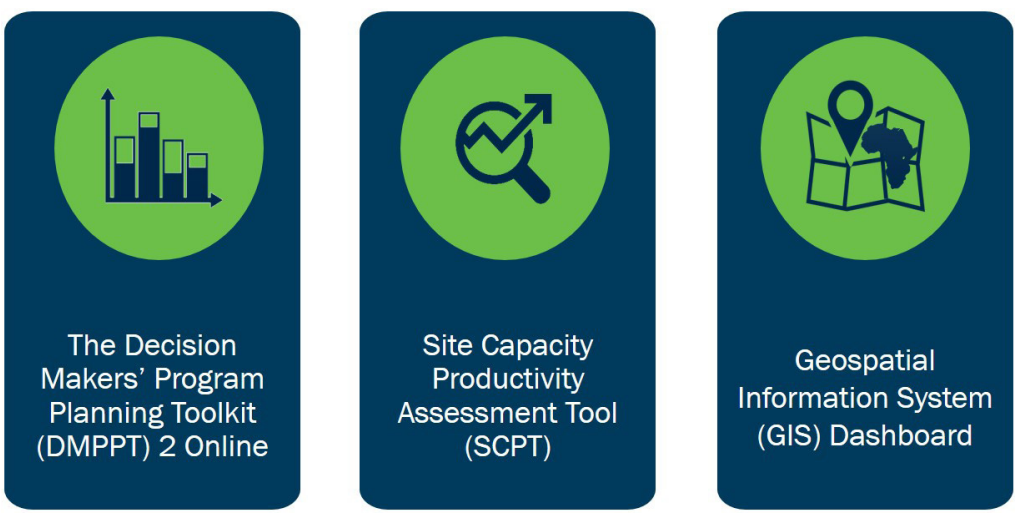

How can I effectively determine VMMC coverage, set targets, and allocate funds to achieve saturation for maximum impact in short-term?

- How can I more effectively plan my program to deliver on time and on target? 
At national, regional, and global levels, targeted workshops have supported extensive dissemination of the suite of VMMC tools and strengthened capacity for their use. ${ }^{7}$

Project SOAR convened 25 national and regional workshops with participants from 10 countries to share the integrated package of VMMC data-fordecision-making tools and strengthen capacity to use them. Participants represented central-level public sector VMMC programs and implementing partners, who engaged in practical, real-world exercises highlighting how the tools can provide data that address issues and challenges met by VMMC programs. Once trained, the participants could cascade the training to sub-national units and service delivery sites.

In addition, SOAR hosted a series of webinars to introduce the tools and train various staff from the U. S. Government, World Health Organization (WHO), UNAIDS, World Bank, Bill \& Melinda Gates Foundation, and ministries of health and implementing partners on the use of the three tools. Finally, SOAR created a short video tutorial describing the operation and features of the DMPPT2 to be included in a Data for Decision Making module for the USAID VMMC Online Training Hub.

\section{Country-level Application of the DMPPT 2}

Project SOAR's extensive stakeholder engagement ensured the DMPPT 2 would meet the needs of intended end-users. The process was documented and published in PLOS ONE. It involved three essential steps: 1) identifying and engaging a wide array of stakeholders from the outset, 2) reaching consensus on key assumptions and analysis plans, and 3) convening data validation meetings with critical stakeholders.

\section{In Mozambique, use of the VMMC suite of tools informed targeted program strategies and data-driven planning that contributed to sustained annual increases in VMMC cov-}

erage, even in provinces with previously the lowest coverage and highest unmet need. ${ }^{8}$

In 2014, Mozambique's national VMMC program adopted a broad site optimization strategy involving site- and community-level strategic planning by implementers; development of site-specific targets; application of the SCPT to assess site-level human resources; and close monitoring of site performance, efficiency, and productivity.

Project SOAR supported the PEPFAR country team and other stakeholders in Mozambique to use data more effectively for decision making, including to focus demand creation activities, set realistic and achievable targets, and allocate program resources to optimize efficiency. With this reorientation in operational strategy, Mozambique experienced a year-on-year increase in VMMC coverage levels due to enhanced capacity and improved program performance.

In two of the geographic regions with lowest VMMC coverage, Manica and Tete provinces, data maps depicting services, sites, and unmet need guided the development of targeted geographic and age-specific demand creation efforts. This new targeted program strategy led to sustained increase in VMMC coverage in the two provinces; Manica province experienced a five-fold increase in coverage from 2014 to 2019 and Tete sustained a 10 -fold increase in coverage over the same period.

Figure 1 Male circumcision coverage in Manica and Tete Provinces, Mozambique

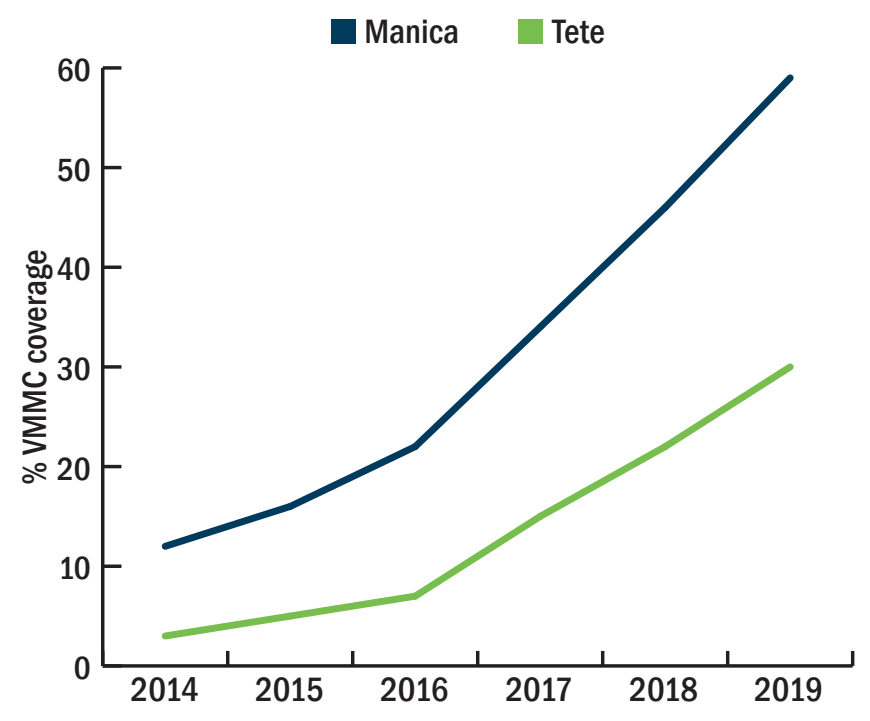




\section{RESEARCH IMPACT SPOTLIGHT}

\section{Sustained Use of Data and Tools Beyond Project SOAR}

Project SOAR has ensured that data-for-decisionmaking tools remain available and sustainable. The DMPPT 2 Online has been widely adopted for use in many VMMC priority countries, and the UNAIDS Reference Group on Estimates, Modelling, and Projections recommended using it to derive better VMMC country data for the annual UNAIDS estimates process, while exploring ways in which to integrate the most commonly used functionalities of the DMPPT 2-producing coverage estimates of circumcision by age and sub-national unit as well as generating VMMC targets by age ${ }^{\text {a }}$-into the models that support the annual UNAIDS estimates process. ${ }^{b}$ Outputs from the estimates process are also used for PEPFAR Country Operational Plans, host country National Strategic Plans, and Global Fund applications. Ensuring annual planning processes are controlled by the national estimates team-a diverse, country-led group including national AIDS agencies, ministries of health, United Nations agencies, U.S. government and other donors and implementers-ensures consistency between national estimates and those used in PEPFAR planning.

\section{SOAR's VMMC tools are recognized at both national and global levels as critical resources to increase the impact of VMMC investments.}

The utility of these tools has been widely recognized at national and global levels. The full VMMC suite of tools, including the DMPPT 2, SCPT, and GIS Dashboard, was recently featured as 1 of 11 Case Studies on Enhancing Uptake of Voluntary Medical Male Circumcision within WHO's 2020 VMMC guidelines. Also, the GIS Dashboard, including its application in South Africa and Tanzania, was cited in PEPFAR's Best Practices for Voluntary Medical Male Circumcision Site Operations: A Service Guide for Site Operations.

Given that data included in the SCPT and GIS Dashboard are routinely collected through country and partner health information systems, Project SOAR worked with country stakeholders to integrate functionality of the tools within existing country data systems. This allows for streamlined access to data, improving quality and timeliness and, ultimately, reducing the need for maintaining multiple systems with similar data. Project SOAR also worked with stakeholders in Malawi and Namibia to pilot the development of a roadmap for integrating functionality within existing systems. In this same process, Project SOAR identified geographic data mapping needs that could then be addressed either through a component akin to the GIS dashboard, or through an existing mapping facility such as those available through the DHIS2.

aThe DMPPT2 Online now has the ability to set individual, age-specific targets at district level. This facility will also be integrated into and supported by the annual UNAIDS estimates process.

bUNAIDS annually publishes country data compiled through: 1) the Global AIDS Response Progress Reports, which address ART, social behaviors, the policy environment, HIV expenditure data, and other indicators measuring progress toward global commitments; and 2) modelled estimates of the number of new infections, people living with HIV and AIDS-related deaths among other variables. This second process will now incorporate circumcision coverage estimates. 


\section{REFERENCES}

1. Project SOAR. 2018. "Modeling the expected costeffectiveness and impact of oral pre-exposure prophylaxis in Uganda and Swaziland," Project SOAR Activity Brief. Washington, DC: Population Council. https://projsoar.org/ wp-content/uploads/2018/04/DREAMS_PrEPMdlng_ ActvtyBrief.pdf

2. Bórquez, A. et al. 2016. "The Incidence Patterns Model to estimate the distribution of new HIV infections in sub-Saharan Africa: Development and validation of a mathematical model," PLoS Medicine 13(9): e1002121. doi: 10.1371/journal.pmed.1002121

3. Project SOAR. 2019. "Oral pre-exposure prophylaxis modeling results: Eswatini," Project SOAR Results Brief. Washington, DC: Population Council. https://projsoar.org/ wp-content/uploads/2019/02/Eswatini_PrEPModeling_ ResBrief.pdf

4. Project SOAR. 2019. "Oral pre-exposure prophylaxis modeling results: Uganda," Project SOAR Results Brief. Washington, DC: Population Council. https://www. projsoar. org/wp-content/uploads/2019/02/Uganda_PrEPModeling ResBrief.pdf

5. Stegman, P. et al. 2019. "Voluntary medical male circumcision to prevent HIV: Modelling age prioritization in Namibia," AIDS and Behavior 23 (Suppl 2): S195-S205. doi: 10.1007/s10461-019-02556-y
6. Tchuenche, M. et al. 2018. "Voluntary medical male circumcision service delivery in South Africa: The economic costs and potential opportunity for private sector involvement," PLoS ONE 13(12): e0208698. doi: 10.1371/ journal.pone.0208698

7. Project SOAR. 2018. "Project SOAR's voluntary medical male circumcision portfolio," Project SOAR Activity Brief. Washington, DC: Population Council. https://www.projsoar. org/wp-content/uploads/2018/06/VMMCPortfolio.pdf

8. Dent, J., N. Gaspar, E. Njeuhmeli, and K. Kripke. 2019. "Age targeting and scale-up of voluntary medical male circumcision in Mozambique," PLoS ONE 14(2): e0211958. doi: 10.1371/journal.pone.0211958

Suggested citation: Project SOAR. 2021. “Improving data access and use for HIV biomedical prevention interventions," Learnings from Project SOAR Synthesis Brief. Washington, D.C.: Population Council.

For more information, please visit Project SOAR.
Project SOAR was a six-year (September 2014-January 2021) cooperative agreement funded by the United States President's Emergency Plan for AIDS Relief and the U. S. Agency for International Development (Agreement No. AID-OAA-A-14-00060). The contents of this brief are the sole responsibility of Project SOAR and Population Council and do not necessarily reflect the views of PEPFAR, USAID, or the United States Government.

Population Council led the Project SOAR consortium in collaboration with Avenir Health, Elizabeth Glaser Pediatric AIDS Foundation, the Johns Hopkins University, Palladium, and The University of North Carolina at Chapel Hill.
Project SOAR/Population Council

4301 Connecticut Avenue, NW, Suite 280

Washington, DC 20008

Tel: +12022379400

e-mail: ProjectSOAR@popcouncil.org projsoar.org

(C) Population Council, April 2021

Cover photo credit: Richard Lord, courtesy of the Population Council 